\title{
ЦИЛИНДРОКЛЕТОЧНАЯ МЕТАПЛАЗИЯ И ПИЩЕВОД БАРРЕТТА: МОРФОЛОГИЧЕСКАЯ НЕОДНОРОДНОСТЬ И ИММУНОГИСТОХИМИЧЕСКИЙ ФЕНОТИП
}

\author{
Л. М. Михалева ${ }^{1,2}$, К. С. Войтковская² , Е. Д. Федоров ${ }^{2,3}$, Н. А. Грачева², А. Е. Бирюков ${ }^{1,2}$, А. В. Шидии-Закруа ${ }^{3}$, М. Ю. Гущин ${ }^{1}$ \\ ${ }^{1}$ Научно-исследовательский институт морфологии человека, Москва, Россия \\ 2 Городская клиническая больница № 31, Москва, Россия \\ ${ }^{3}$ Российский национальный исследовательский медицинский университет имени Н. И. Пирогова, Москва, Россия
}

\begin{abstract}
Пищевод Барретта (ПБ), или доказанная морфологически кишечная метаплазия слизистой оболочки дистального отдела пищевода, является облигатным предраковым заболеванием, которое развивается как осложнение гастроэзофагеальной реслюксной болезни (ГЭРБ). Цель исследования - выполнить сравнительный морфологический анализ биопсированных фрагментов ПБ, ЦМ на расстоянии < 1 см и > 1 см от гастроэзофагеального перехода (ГЭП), а также провести иммуногистохимическое исследование фрагментов с ПБ и ЦМ > 1 см от ГЭП при наличии и отсутствии дисплазии. В исследование вошли 92 пациента с ГЭРБ: 42 пациента с ПБ, 24 пациента с ЦМ > 1 см от ГЭП (СОМ1,5 до С13М14) и 26 пациентов с ЦМ < 1 см от ГЭП (СОМО,3-0,8). При сравнительном морфологическом анализе наличие реактивных изменений эпителия было связано с тяжестью эзофагита во всех группах. Реактивные изменения эпителия выявляли достоверно чаще при ПБ по сравнению с ЦМ > 1 см от ГЭП ( $<<0,05$ при использовании критерия Манна-Уитни). При ПБ в восьми наблюдениях (19,05\%) выявлена low-grade дисплазия. В одном наблюдении ЦМ > 1 см от ГЭП (4,2\%) выявлена high-grade дисплазия на фоне кардиальной метаплазии с иммуногистохимическими признаками субморфологической энтерализации. При иммуногистохимическом исследовании с маркерами желудочной и кишечной дифференцировки признаки субморфологической энтерализации выявлены во всех фрагментах пищевода с кардиальной метаплазией и у пациентов с ПБ в зонах с отсутствием бокаловидных клеток.
\end{abstract}

Ключевые слова: цилиндроклеточная метаплазия пищевода, пищевод Барретта, low-grade и high-grade дисплазия, канцерогенез

Информация о вкладе авторов: Л. М. Михалева - планирование и руководство исследованием, предоставление материально-технической базы для проведения исследования, анализ результатов; К. С. Войтковская - анализ литературы, сбор, анализ и интерпретация данных, подготовка микрофотографий и рукописи; Е. Д. Федоров - клиническое обследование пациентов, выполнение ЭГДС с взятием биопсийного материала, анализ и обобщение полученных данных; А. В. Шидии-Закруа - клиническое обследование пациентов, выполнение ЭГДС с взятием биопсийного материала; А. Е. Бирюков - исследование и анализ биопсийного материала; Н. А. Грачева — исследование и анализ биопсийного материала; М. Ю. Гущин - сбор и анализ литературы.

Соблюдение этических стандартов: все пациенты подписали добровольное информированное согласие.

$\triangle$ Для корреспонденции: Людмила Михайловна Михалева

ул. Цюрупы, д. 3, г. Москва, 117418; mikhalevalm@yandex.ru

Статья получена: 03.12.2019 Статья принята к печати: 18.12.2019 Опубликована онлайн: 25.12.2019

DOI: $10.24075 /$ vrgmu.2019.086

\section{COLUMNAR METAPLASIA AND BARRETT'S ESOPHAGUS: MORPHOLOGICAL HETEROGENEITY AND IMMUNOHISTOCHEMICAL PHENOTYPE}

Mikhaleva LM ${ }^{1,2}$, Voytkovskaya KS², Fedorov ED ${ }^{2,3}$, Gracheva NA², Birukov AE ${ }^{1,2}$, Shidiy-Zakrua AV³, Guschin MY1

${ }^{1}$ Research Institute of Human Morphology, Moscow, Russia

${ }^{2}$ City Clinical Hospital № 31, Moscow, Russia

${ }^{3}$ Pirogov Russian National Research Medical University, Moscow, Russia

Barrett's esophagus (BE) is a pathologically confirmed intestinal metaplasia (CM) of the distal esophagus. BE is recognized as a potential complication of gastroesophageal reflux disease (GERD) and a premalignant condition with a high risk of neoplastic progression. The aim of this study was to compare the morphology of biopsied BE segments and CM segments extending $<1 \mathrm{~cm}$ and $>1 \mathrm{~cm}$ above the gastroesophageal junction (GEJ), as well as to perform the immunohistochemical analysis of biopsies with BE and $\mathrm{CM}>1 \mathrm{~cm}$ above GEJ with or without dysplasia. The study recruited 92 patients with GERD: 42 patients with BE, 24 patients with $\mathrm{CM}>1 \mathrm{~cm}$ above GEJ (COM1.5-C13M14) and 26 patients with $\mathrm{CM}<1 \mathrm{~cm}$ above GEJ (COMO.3-0.8). Comparative analysis of tissue morphology revealed an association between the reactive changes in the epithelium and the severity of esophagitis in all groups. Reactive changes were detected significantly more often in BE segments than in CM segments $>1 \mathrm{~cm}$ (Mann-Whitney $\mathrm{U}, p<0.05$ ). Eight patients with BE (19.05\%) were found to have lowgrade dysplasia. One patient with $\mathrm{CM}>1 \mathrm{~cm}$ above GEJ (4.2\%) had high-grade dysplasia with cardiac-type metaplasia and immunohistochemical features of submorphological enteralization. Immunohistochemical testing for the intestinal and gastric markers of cell differentiation revealed the signs of submorphological enteralisation in all esophageal specimens with cardiac and fundic type metaplasia and in the specimens with BE in the areas lacking goblet cells.

Keywords: columnar metaplasia, Barrett's esophagus, low-grade dysplasia, high-grade dysplasia, carcinogenesis

Author contribution: Mikhaleva LM — planned and supervised the study; provided equipment for the study; analyzed the obtained results; Voytkovskaya KS — analyzed the literature; collected, analyzed and interpreted the obtained data; processed microphotographs and wrote the manuscript; Fedorov ED — examined the patients; performed EGD and biopsy; analyzed and summarized the data; Shidiy-Zakrua AV — examined the patients, performed EGD and biopsy; Birukov AE — and Gracheva NA — performed pathological examination; Guschin MY — analyzed the literature.

Compliance with ethical standards: all patients gave written informed consent.

Correspondence should be addressed: Ludmila M. Mikhaleva

Tsyuryupy 3, Moscow, 117418; mikhalevalm@yandex.ru

Received: 03.12.2019 Accepted: 18.12.2019 Published online: 25.12.2019

DOI: $10.24075 /$ brsmu.2019.086 
Пищевод Барретта (ПБ), под которым подразумевают доказанную эндоскопически и морфологически цилиндроклеточную кишечную метаплазию слизистой оболочки дистального отдела пищевода, является облигатным предраковым заболеванием, которое развивается как осложнение гастроэзофагеальной рефлюксной болезни (ГЭРБ) у ряда пациентов. В настоящее время в мире нет единого определения термина «пищевод Барретта». Это связано с целым рядом причин, в том числе с большой разнородностью патогистологических изменений участка трансформации эпителия в дистальном отделе пищевода с наличием нескольких типов метаплазии и их мозаичным сочетанием даже в пределах одного биопсированного фрагмента. В то время как Американская ассоциация гастроэнтерологов настаивает на том, что для постановки диагноза «пищевод Барретта» обязательно выявление кишечной метаплазии в биоптатах из участка метаплазии слизистой оболочки дистального отдела пищевода [1], Британское общество гастроэнтерологов (BSG) называет пищеводом Барретта любой тип цилиндроклеточной метаплазии (ЦМ), распространяющийся выше 1 см от уровня гастроэзофрагеального перехода (ГЭП) [2]. По Международному консенсусу ВОВ САТ (2015), пищеводом Барретта называют любой тип ЦМ слизистой оболочки дистального отдела пищевода, но с обязательным указанием в гистологическом заключении типа метаплазии [3].

Определение ПБ, данное BSG, нашло немало сторонников и стало достаточно популярным, но не изменило биологической сути заболевания. Вот почему биоптаты, взятые на расстоянии < 1 см от ГЭП, нуждаются в особенно тщательном сопоставлении эндоскопической картины и морфологических данных [4].

В дистальном отделе пищевода выделяют кардиальную, фундальную и кишечную метаплазию слизистой оболочки. Кардиальную метаплазию чаще можно встретить в коротком сегменте ПБ, это наиболее раннее метапластическое изменение пищевода в условиях ГЭРБ. При кардиальной метаплазии эпителий соответствует фовеолярному. При фундальной метаплазии добавляются элементы, характерные для слизистой оболочки тела желудка - главные и париетальные клетки. При кишечной метаплазии бокаловидные клетки в железах перемежаются с клетками фовеолярного типа. Иногда в биоптатах ПБ с кишечной метаплазией можно найти клетки Панета [5].

У одного и того же пациента в биоптатах пищевода можно встретить различные типы ЦМ. При этом, как правило, кишечную метаплазию выявляют более проксимально в сегменте ПБ, а кардиальную и фундальную метаплазию - ближе к ГЭП. Обнаружено, что кишечная метаплазия встречается в два раза чаще в биопсиях из проксимальных отделов ПБ по сравнению с биопсиями, взятыми из пищеводно-желудочного соединения [6].

Патогенез ЦМ пищевода в настоящее время связывают либо с трансдифференцировкой многослойного плоского эпителия, либо с транскоммитированием клетокпредшественников под действием рефлюкса в условиях заживления повреждения [7].

Частота обнаружения бокаловидных клеток связана с градиентом рН вдоль сегмента ПБ: чем ниже рН (т. е., чем ближе к ГЭП), тем реже и в меньшем количестве выявляют бокаловидные клетки. Это связано, по всей видимости, с градиентом растворимости желчных кислот, который обусловлен значением pH: при низком рН ближе к ГЭП растворимость билиарных кислот минимальна, при средних значениях рН в проксимальном сегменте
ПБ растворимость билиарных кислот повышается [8]. В исследовании на крысах показана патогенетическая роль желчных кислот в развитии ПБ с кишечной метаплазией и экспрессией CDX2 и MUC2 [9].

Показано также, что кардиальные клетки могут подвергаться ранней энтерализации, т. е., сохраняя морфологию кардиальных клеток, экспрессировать маркеры кишечной диффференцировки: виллин и CDX2 [10].

Ведутся дискуссии о том, является ли роль бокаловидных клеток исключительной при канцерогенезе в дистальном отделе пищевода или другие типы ЦМ тоже могут вносить свой вклад в развитие дисплазии и аденокарциномы пищевода. Эпидемиологические данные указывают на то, что частота опухолевой прогрессии выше у пациентов с кишечной метаплазией $(0,38 \%$ в год) по сравнению с пациентами, у которых в биопсиях не выявлена кишечная метаплазия (0,07\% в год) [11]. В другом эпидемиологическом исследовании, где длительность динамического наблюдения за пациентами составила 8-20 лет, такой закономерности не найдено [12]. Показано, что более чем у $70 \%$ пациентов с небольшими по размерам (до 2 см) аденокарциномами пищевода предшествующим изменением была цилиндрическая метаплазия пищевода, но не кишечного, а кардиального типа, которую обнаруживали вокруг участков аденокарциномы в биопсиях пищевода [13]. Эти данные подтверждены и другими авторами [14], обнаружившими желудочный фенотип (экспрессию MUC5A и MUC6) при миниатюрных размерах опухоли (< 5 мм). Кроме того, малигнизация может происходить в эпителии ПБ кардиального типа без бокаловидных клеток и без экспрессии CDX2 [15]. В то же время высокая плотность бокаловидных клеток может играть защитную роль и снижает риск развития аденокарциномы [16, 17]. Вероятно, В дистальном отделе пищевода существует два независимых пути канцерогенеза: фовеолярный и кишечный, т. е. с участием метаплазии как желудочного, так и кишечного типа [18-20]. Определить тип канцерогенеза можно при помощи иммуногистохимического исследования на основании экспрессии желудочных (MUC1, MUC5A и MUC6) и кишечных (MUC 2, CD10, CDX2, виллин и др.) маркеров.

Протяженность сегмента метаплазии дистального отдела пищевода > 1 см от уровня ГЭП, которая по определению BSG необходима для диагностики ПБ, все шире используемый, но довольно условный критерий. Данные об участии в канцерогенезе пищевода ЦМ длиной $<1$ см по-прежнему противоречивы.

Целью нашего исследования было провести сравнительный морфологический анализ ЦМ пищевода, протяженностью < 1 см от уровня ГЭП (так называемого ультракороткого сегмента ПБ), классического ПБ с наличием бокаловидных клеток и ЦМ > 1 см от ГЭП с определением относительной частоты различных типов метаплазии, частоты реактивных изменений эпителия (так называемой неопределенной степени дисплазии) и дисплазии метаплазированного эпителия пищевода, а также выполнить иммуногистохимическое исследование фрагментов ПБ с разными типами метаплазии при наличии или отсутствии дисплазии.

\section{ПАЦИЕНТЫ И МЕТОДЫ}

Исследование проводили на базе Городской клинической больницы № 31 в период с 1 января 2018 г. по 1 сентября 2019 г. В исследование были включены пациенты с 
ГЭРБ, проходившие обследование в ГКБ № 31. Критерии включения пациентов: эндоскопически обнаруженные участки цилиндроклеточной метаплазии пищевода любой протяженности, подтвержденные затем морфологически. Материалом исследования послужили биопсированные фрагменты слизистой оболочки дистального отдела пищевода, полученные при ЭГДС у 92 пациентов с ГЭРБ: у 42 пациентов с ПБ, 24 пациентов с ЦМ > 1 см от ГЭП (с протяженностью участков метаплазии от С0M1,5 до C13M14) и 26 пациентов с эндоскопическими признаками ЦМ < 1 см От ГЭП (СОМО,3-0,8). Критерии исключения: отсутствие цилиндроклеточной метаплазии в биоптатах при морфологическом исследовании; отсутствие в биоптатах дериватов пищевода (многослойный плоский эпителий, железы собственной пластинки слизистой пищевода и их протоки), особенно при ультракоротком сегменте ПБ, что не позволяло с уверенностью утверждать, что материал получен из пищевода, а не из желудка. В группе ЦМ < 1 см от ГЭП средний возраст пациентов составил 55,50 \pm 1,10 лет (22-82 года), среди них 11 мужчин (средний возраст $50,09 \pm 18,03)$ и 15 женщин $(59,47 \pm 14,57)$, соотношение мужчин и женщин $1: 1,36$. Группа пациентов с ЦМ $>1$ см от ГЭП представлена 24 пациентами в возрасте 19-94 лет (средний возраст 52,21 \pm 18,00 лет), среди них 7 мужчин (средний возраст $47 \pm 20,05)$ и 17 женщин (средний возраст 53,5 $\pm 17,47$ ), соотношение мужчин и женщин приблизительно $1: 2,4$. Группа пациентов с ПБ представлена 42 пациентами в возрасте 19-93 лет (средний возраст 61,80 $\pm 16,33$ ), из них 29 мужчин (средний возраст 54,47 \pm 21,79) и 13 женщин (69,23 \pm 13,57), соотношение мужчин и женщин 2,23 : 1. Как тенденция у пациентов с ЦМ < 1 см и > 1 см от ГЭП прослеживается более молодой возраст по сравнению с пациентами с ПБ, однако статистически значимые различия не выявлены. Как в группах ЦМ пищевода < 1 см, так и при ЦМ > 1 см от ГЭП и при ПБ средний возраст у мужчин был несколько меньше, чем у женщин, поскольку мужской пол - один из факторов риска развития ГЭРБ.

Фоновыми заболеваниями, которые предрасполагали к развитию ЦМ дистального отдела пищевода и ПБ, являлись аксиальная хиатальная грыжа и недостаточность кардии. Так, у 14 из 26 пациентов (53,8\%) с эндоскопическими признаками ЦМ < 1 см выявлена эндоскопическая картина аксиальной хиатальной грыжи, еще у 3 (11,5\%) признаки недостаточности кардии при отсутствии хиатальной грыжи. У 11 пациентов (45,83\%) с ЦМ > 1 см от ГЭП при ЭГДС найдена хиатальная грыжа, а у 3 (12,5\%) —

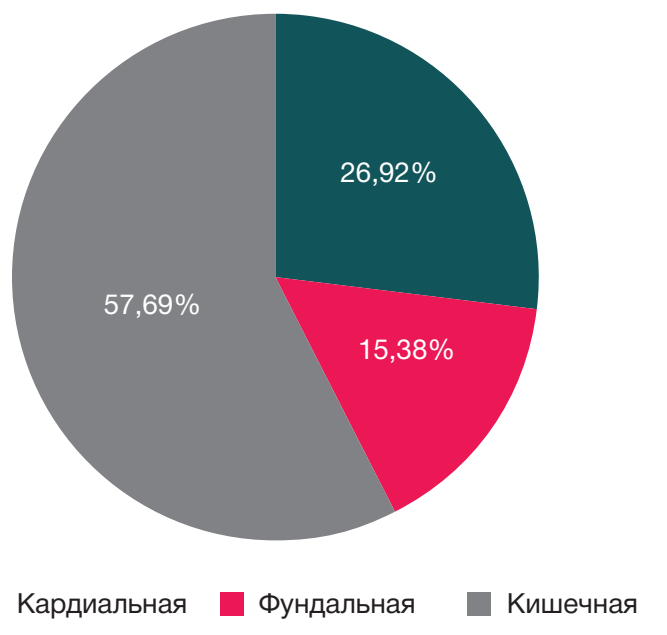

Рис. 1. Распространенность разных типов метаплазии при ЦМ < 1 см над ГЭП недостаточность кардии. У 18 пациентов $(42,86 \%)$ с ПБ выявлены эндоскопические признаки хиатальной грыжи, еще у $6(14,29 \%)$ признаки недостаточности кардии.

Биопсированные фрагменты пищевода после стандартной обработки и получения гистологических срезов окрашивали гематоксилином и эозином, а также Шиффреактивом в сочетании с альциановым синим. Вторая окраска помогает достоверно выявить бокаловидные клетки, а также различить бокаловидные и дистрофически измененные псевдобокаловидные клетки. Псевдобокаловидные клетки выявлены в 88,04\% всех наблюдений.

В наблюдениях с кишечной метаплазией проводили морфометрический подсчет плотности бокаловидных клеток в железах: при наличии бокаловидных клеток, занимающих < 5\% от всех эпителиоцитов в железах, пациентов относили к подгруппе с единичными бокаловидными клетками; если бокаловидные клетки занимали от 5 до 50\%, пациентов относили к подгруппе с низкой плотностью бокаловидных клеток; когда бокаловидные клетки занимали > 50\%, пациентов относили к подгруппе с высокой плотностью бокаловидных клеток.

В биопсированных фрагментах у всех пациентов с ЦМ < 1 см определяли структуры, характерные для слизистой оболочки пищевода: многослойный плоский эпителий - у 20 пациентов (76,92\%), железы пищевода - у 19 пациентов (73,07\%), выводные протоки желез слизистой пищевода - у 5 пациентов (19,23\%). Выявление этих морфологических структур помогало определить, что материал взят из дистального отдела пищевода, а не из желудка. Фрагменты, в которых отсутствовали характерные структуры слизистой пищевода, не учитывали при анализе материала.

В 24 наблюдениях ПБ и ЦМ > 1 см от ГЭП проводили иммуногистохимическое исследование с моноклональными антителами к MUC1 (1: 100, Ventana; Roche), MUC2 (1 : 125, Ventana; Roche), MUC5A (1: 250, Ventana; Roche), MUC6 (готовые антитела Ventana; Roche), CDX2 (1 : 125, Ventana; Roche) и виллину (готовые антитела; Leica).

\section{РЕЗУЛЬТАТЫ ИССЛЕДОВАНИЯ}

При ЦМ < 1 см от ГЭП выявляли различные типы метаплазии: кардиальную, фундальную и кишечную. В группе пациентов с ЦМ пищевода < 1 см (рис. 1) только кардиальная метаплазия была определена в 7 случаях (26,92\%), фундальная — в 4 случаях (15,38\%).

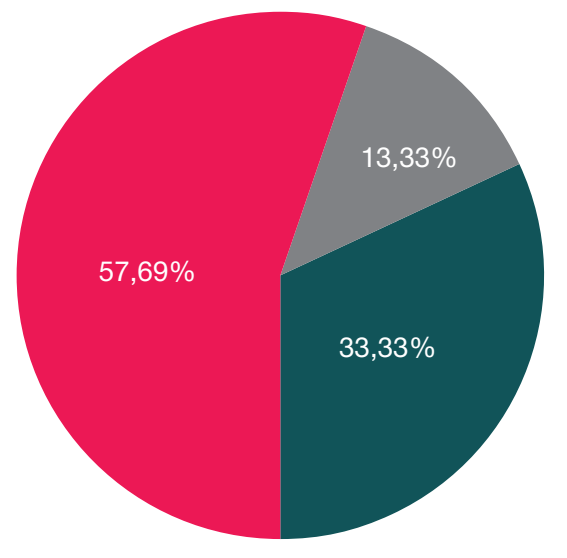

Единичные

Низкое

Высокое

Рис. 2. Относительное число бокаловидных клеток при ЦМ < 1 см от ГЭП 
У 15 пациентов $(57,69 \%)$ выявлена кишечная метаплазия с разной относительной плотностью бокаловидных клеток (рис. 2): у 5 пациентов (33,33\%) наблюдали единичные бокаловидные клетки, у 8 пациентов (53,33\%) - низкую плотность бокаловидных клеток (от 10 до 49\%), у 2 (13,33\%) - высокую плотность бокаловидных клеток (50 и 70\% бокаловидных клеток в железах). Мозаичное сочетание трех типов метаплазии обнаружено в образцах биопсии 6 пациентов (23,07\%). Во всех биопсированных фррагментах дистального отдела пищевода фоновым процессом был эзофагит, при этом воспалительная инфильтрация была умеренной (в 69,23\% случаев) и выраженной (в 30,77\% случаев), с наличием в образцах биопсии у 8 пациентов $(30,77 \%)$ эрозий слизистой оболочки метаплазированного пищевода и язвенного дефекта у 1 пациента. У 9 пациентов $(34,62 \%)$ на фоне выраженного воспаления выявлены реактивные изменения эпителия желез (так называемая дисплазия неопределенной степени), характеризующиеся иррегулярным расположением и сближенностью желез, их угловатостью, легким укрупнением и гиперхромией части ядер, единичными митозами. В 2 наблюдениях реактивные изменения эпителия были обнаружены при кардиальной метаплазии слизистой пищевода, в 6 случаях - при кишечной метаплазии с низким содержанием бокаловидных клеток, в 1 - с высоким содержанием бокаловидных клеток. Ни в одном из фрагментов пищевода при ЦМ < 1 см не обнаружена lowgrade или high-grade дисплазия.

В группе ЦМ > 1 см от ГЭП кардиальная метаплазия выявлена в 14 из 24 наблюдений (58,33\%) и фундальная - в 10 наблюдениях $(41,67 \%)$. В группу пациентов с ПБ вошло 42 пациента с кишечной метаплазией. Если объединить группы ЦМ > 1 см От ГЭП и ПБ, соотношение типов метаплазии получится следующим (рис. 3): кардиальная метаплазия - 14 наблюдений из 66 (21,21\%), фундальная 10 (15,15\%), кишечная - 42 (63,64\%), мозаичное сочетание трех типов метаплазии обнаружено в образцах биопсии 8 пациентов с ПБ (12,12\%). В группе пациентов с ПБ (42 наблюдения с кишечной метаплазией; рис. 4) единичные бокаловидные клетки выявлены в 8 случаях (18,18\%), низкая плотность бокаловидных клеток - в 15 случаях (43,09\%), высокая плотность бокаловидных клеток - в 21 случае $(47,73 \%)$.

Таким образом, кишечную метаплазию в образцах биопсии объединенной группы пациентов с ЦМ > 1 см от ГЭП и ПБ выявляли несколько чаще, чем при ЦМ дистального

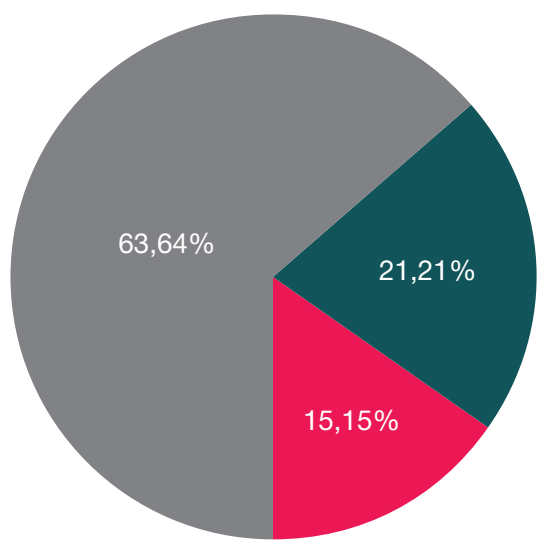

Кардиальная

Фундальная

Кишечная

Рис. 3. Распространенность разных типов ЦМ при протяженности сегмента > 1 см от ГЭП, включая ПБ отдела пищевода на расстоянии < 1 см от ГЭП (в 63,64 и 57,69\% наблюдений соответственно; рис. 5). При этом при ПБ в 3,5 раза чаще, чем при ЦМ < 1 см от ГЭП обнаружена высокая плотность бокаловидных клеток (рис. 6).

Признаки эзофагита с умеренной воспалительной инфильтрацией лимфоцитами и плазмоцитами были определены у 16 пациентов с цилиндроклеточной метаплазией > 1 см от ГЭП (66,67\%), а признаки выраженного воспаления - у 8 пациентов (33,33\%), у 11 пациентов выявлены эрозии метаплазированного пищевода $(45,83 \%)$, из них у 1 пациента обнаружены участки изъязвления. Реактивные изменения выявлены у 5 пациентов с цилиндроклеточной метаплазией (20,83\%): у 3 - с кардиальной метаплазией и у 2 - с фундальной. У одного пациента выявлена highgrade дисплазия метаплазированного сегмента на фоне кардиальной метаплазии (4,2\%).

Признаки эзосагита с умеренной воспалительной инфильтрацией лимфоцитами и плазмоцитами найдены у 18 пациентов с ПБ (42,86\%), а признаки выраженного воспаления - у 26 пациентов $(57,14 \%) ;$ у 27 пациентов выявлены эрозии метаплазированного пищевода (64,29\%), из них у 4 пациентов - участки изъязвления. У пациентов с ПБ эрозии обнаруживали в 2,1 раза чаще, чем при ЦМ < 1 см от ГЭП, и в 1,4 раза чаще, чем у пациентов с ЦМ $>1$ см от ГЭП. При этом реактивные изменения эпителия наблюдали у 15 пациентов (35,71\%). Таким образом, реактивные изменения эпителия желез определяли почти одинаково часто при ПБ и ЦМ < 1 см от ГЭП и в 1,7 раза реже при ЦМ > 1 см от ГЭП без кишечной метаплазии. Реактивные изменения эпителия были обнаружены достоверно чаще в группе пациентов с ПБ по сравнению с группой ЦМ > 1 см От ГЭП (при использовании критерия Манна-Уитни, $p<0,05)$. Реактивные изменения выявляли одинаково часто при различной плотности бокаловидных клеток (в наблюдениях с единичными бокаловидными клетками, с низким числом бокаловидных клеток и высоким числом бокаловидных клеток). Реактивные изменения во всех случаях были связаны с тяжестью воспаления (при использовании критерия Манна-Уитни, $p<0,05)$.

При ЦМ < 1 см от ГЭП наблюдения c low-grade $и$ high-grade дисплазией отсутствовали, при ПБ low-grade дисплазия (рис. 7А) диагностирована в 8 наблюдениях (19,05 \%), из них 6 случаев - с высокой плотностью бокаловидных клеток и 2 - с единичными бокаловидными клетками, а при ЦМ > 1 см от ГЭП выявлен один случай с high-grade дисплазией (4,2\% наблюдений; рис. 7Б).

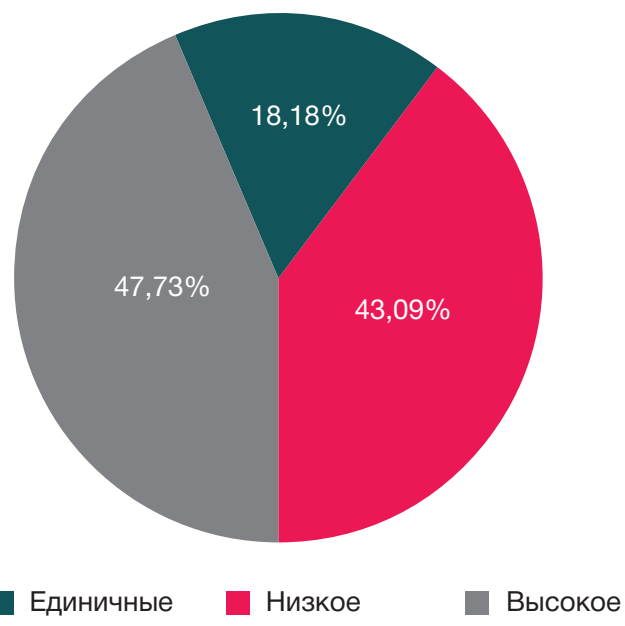

Рис. 4. Относительное число бокаловидных клеток при ПБ 
В 8 наблюдениях ЦМ > 1 см от ГЭП и 16 наблюдениях ПБ произведено иммуногистохимическое исследование с маркерами кишечной (MUC2, CDX2, виллин) и желудочной (MUC1, MUC5A, MUC6) дифференцировки. При кардиальной и фундальной метаплазии слизистой оболочки пищевода наблюдали насыщенную диффузную экспрессию MUC5A в цитоплазме поверхностного эпителия, неяркую диффузную экспрессию MUC1 в цитоплазме поверхностного эпителия, диффузную экспрессию MUC1, MUC5A и MUC6 в цитоплазме эпителия желез. Экспрессия MUC2 при кардиальной и фундальной метаплазии отсутствовала у всех пациентов. Очаговая экспрессия CDX2 при кардиальной и фундальной метаплазии выявлена у 5 пациентов (от единичных клеток до 30\% площади фрагментов), а экспрессия виллина - во всех 8 случаях и занимала от 15-20 до 80\% эпителия желез. Экспрессия кишечных маркеров (CDX2 и виллина) при кардиальной и фундальной метаплазии пищевода служит признаком субморфологической энтерализации. При ПБ с кишечной метаплазией во всех 16 случаях выявляли экспрессию кишечных маркеров (MUC 2, CDX2, виллин), которая в количественном отношении зависела от плотности бокаловидных клеток: цитоплазматическая экспрессия MUC2 занимала 10-50\% клеток, ядерная экспрессия CDX2 составляла 10-90\% эпителиальных клеток, включая цилиндрический эпителий желез, имеющий морфологию кардиального типа, цитоплазматическая экспрессия виллина обнаружена в 70-100\% клеток эпителия желез. Поскольку бокаловидные клетки в метаплазированном эпителии ПБ располагаются между клетками фовеолярного типа, при кишечной метаплазии также выявляется экспрессия желудочных маркеров - MUC1, MUC5A и MUC6, которая, как правило, несколько слабее, чем при кардиальной метаплазии и занимает меньшую протяженность.

При иммуногистохимическом исследовании фррагментов ПБ c low-grade дисплазией диагностирована выраженная экспрессия MUC2, CDX2 и виллина, а также слабая экспрессия MUC1, MUC5A и MUC6, в 1 наблюдении high-grade дисплазии на фоне кардиальной метаплазии выявлена экспрессия MUC1, MUC5A, MUC6 и виллина при негативной экспрессии MUC2 и CDX2.

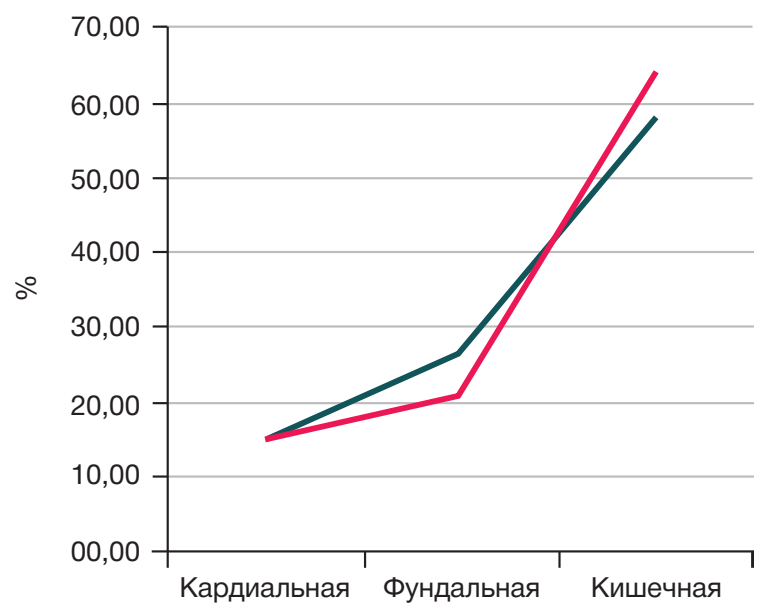

Цилиндроклеточная метаплазия

$<1$ см от ГЭП

Цилиндроклеточная метаплазия

$>1$ см От ГЭП

Рис. 5. Относительная частота различных типов метаплазии при ЦМ < 1 см и $>1$ см от ГЭП

\section{ОБСУЖДЕНИЕ РЕЗУЛЬТАТОВ}

В исследовании проведен сравнительный морсологический анализ биоптатов ЦМ < 1 см от ГЭП, ЦМ > 1 см от ГЭП и ПБ. Интересно, что половой состав выборки пациентов с ЦМ > 1 см От ГЭП и ПБ отличался почти зеркально: соотношение мужчин и женщин при ЦМ > 1 см от ГЭП составило $1: 2,4$, а при ПБ - 2,23: 1. Средний возраст у мужчин во всех группах был несколько меньше, чем у женщин, поскольку мужской пол - один из факторов риска развития ГЭРБ. Считается, что кардиальная метаплазия является самым ранним типом ЦМ пищевода при ГЭРБ. И действительно, в нашем исследовании только кардиальная метаплазия выявлялась несколько чаще при ЦМ < 1 см (26,92\%), чем при длине метаплазированного сегмента C0M1,5-C13M14 (21,21\%). При длине метаплазированного сегмента пищевода > 1 см от ГЭП бокаловидные клетки обнаруживались чаще: при этом высокую плотность бокаловидных клеток при ПБ наблюдали в 3,5 раза чаще, чем при ЦМ < 1 см. По литературным данным, частота выявления бокаловидных клеток увеличивается с возрастанием длины метаплазированного сегмента пищевода [6]. Целью нашего дальнейшего исследования станет выявление взаимосвязи между длиной сегмента ПБ, частотой обнаружения бокаловидных клеток и их плотностью.

Реактивные изменения эпителия были обнаружены достоверно чаще в группе пациентов с ПБ по сравнению с группой > 1 см от ГЭП и были связаны с тяжестью воспаления (при использовании критерия Манна-Уитни, $p<0,05)$. При ПБ дисплазию выявляли значительно чаще (в 19,05\% наблюдений), чем при ЦМ > 1 см от ГЭП (4,2\% наблюдений). В наблюдениях c low-grade дисплазией при ПБ выявляли выраженную экспрессию кишечных (MUC2, CDX2 и виллина) и более слабую экспрессию желудочных (MUC1, MUC5A и MUC6) маркеров, что соответствует смешанному фенотипу с преобладанием кишечного [13, 14, 18-20]. В единственном наблюдении high-grade дисплазии при ЦМ > 1 см от ГЭП найдены яркая экспрессия MUC1, MUC5A, MUC6 и виллина и отрицательная экспрессия MUC2 и CDX2, что свидетельствует о кардиальном фенотипе. Таким образом, мы наблюдали как кишечный

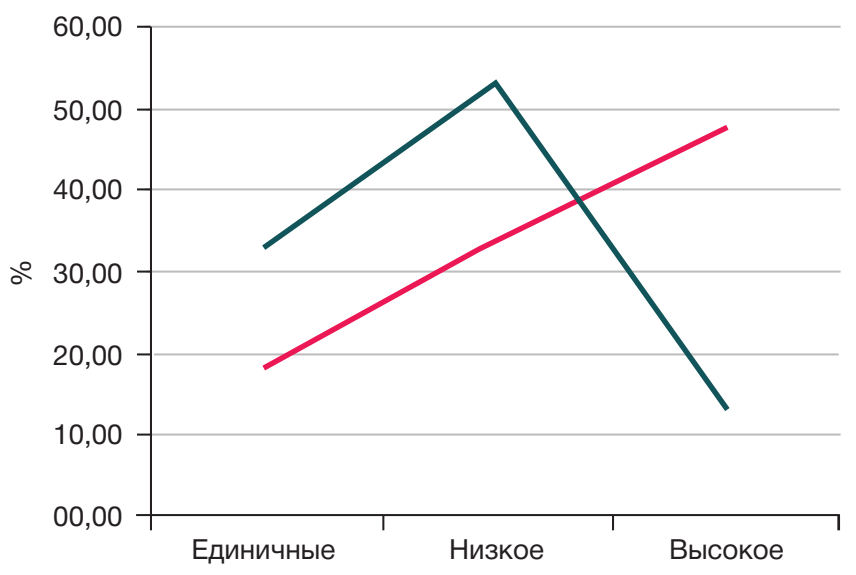

Относительное число бокаловидных клеток при ЦМ < 1 см от ГЭП

Относительное число бокаловидных клеток при пищеводе Барретта

Рис. 6. Относительное число бокаловидных клеток при ЦМ < 1 см от ГЭП и при ПБ 
A

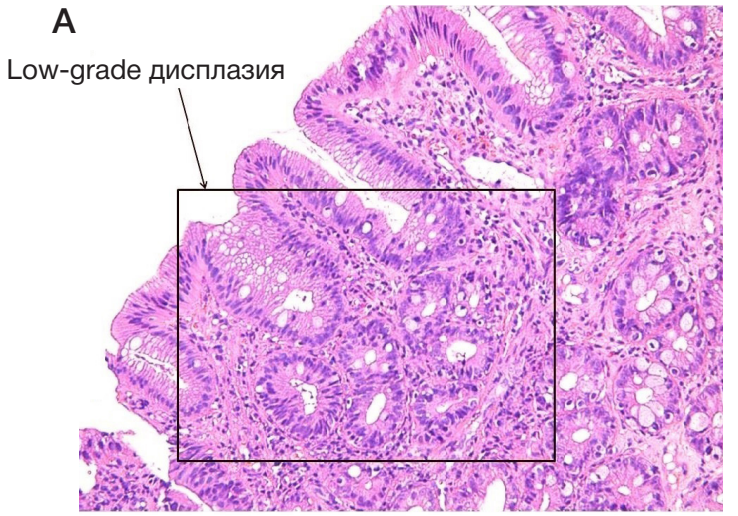

Б

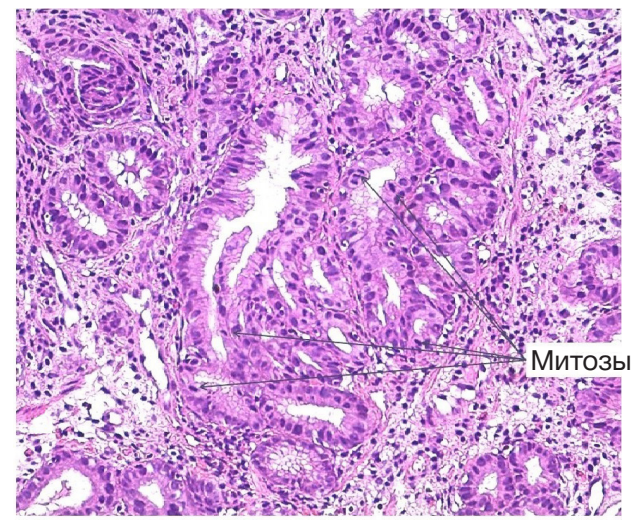

Рис. 7. A. Low-grade дисплазия пищевода при ПБ. Б. High-grade дисплазия пищевода при ЦМ > 1 см от ГЭП Окраска гематоксилином и эозином; увеличение $\times 200$

(при ПБ), так и фовеолярный (при ЦМ пищевода > 1 см от ГЭП) пути канцерогенеза, однако кишечный путь в нашей выборке по распространенности преобладал, несмотря на миниатюрные размеры участков дисплазии (до 2 мм).

Предполагается, что высокая плотность бокаловидных клеток может иметь протективное значение: при высокой плотности бокаловидных клеток дисплазия встречается реже $[16,17]$. В нашей выборке low-grade дисплазия выявлена в 6 из 8 случаев (75\%) при высокой плотности бокаловидных клеток в окружающей слизистой пищевода. Это может быть связано с небольшим числом наблюдений low-grade дисплазии в нашем исследовании. Необходимо продолжить исследование для более объективной оценки морфологических особенностей ПБ при наличии дисплазии, а также определить группу риска пациентов в отношении ее развития.

\section{ВЫВОДЫ}

Для оценки образцов биопсии из ультракороткого сегмента ПБ особое значение имеет прицельный и точный забор материала. Этот диагноз можно установить только при выявлении в биоптатах морфологически четко определяемых структур метаплазированного эпителия ПБ с наличием дериватов пищевода (многослойного плоского эпителия, выводных протоков и желез слизистой). При сравнительном морфологическом анализе реактивные изменения эпителия выявляли достоверно чаще при ПБ по сравнению с группой ЦМ $>1$ см От ГЭП $(p<0,05$ при использовании критерия Манна-Уитни), а в группе ЦМ < 1 см От ГЭП - чаще при наличии бокаловидных клеток. Наличие реактивных изменений эпителия было связано с тяжестью эзофагита. Истинная дисплазия выявлена в 8 наблюдениях с ПБ (low-grade дисплазия 19,05\% наблюдений) и в 1 наблюдении ЦМ пищевода $>1$ см от ГЭП (high-grade дисплазия - 4,2\% наблюдений). При иммуногистохимическом исследовании с маркерами желудочной и кишечной дифференцировки признаки субморфологической энтерализации выявлены во всех фрагментах пищевода с кардиальной и фундальной метаплазией и у пациентов с ПБ в зонах с отсутствием бокаловидных клеток.

\section{Литература}

1. Shaheen NJ, Falk GW, lyer PG, Gerson LB. ACG clinical guideline: diagnosis and management of Barrett's esophagus. Am J Gastroenterol. 2016; 111 (1): 30-50. PubMed PMID: 26526079.

2. Fitzgerald RC, di Pietro $M$, Ragunath $\mathrm{K}$, Ang $\mathrm{Y}$, et al. British Society of Gastroenterology guidelines on the diagnosis and management of Barrett's oesophagus. Gut. 2014; (63): 7-42. PubMed PMID: 24165758.

3. Bennett C, Moayyedi P, Corley DA, DeCaestecker $J$ et al. BOB CAT: A Large-Scale Review and Delphi Consensus for Management of Barrett's Esophagus With No Dysplasia, Indefinite for, or Low-Grade Dysplasia. Am J Gastroenterol. 2015; (110): 662-82. PubMed PMID: 25869390.

4. Montgomery EA, Canto MI, Srivastava A. Evaluation and reporting of biopsies from the columnar-lined esophagus and gastroesophageal junction (GEJ). Ann Diagn Pathol. 2019; (39): 111-7. PubMed PMID: 30802810.

5. Biswas S, Quante M, Leedham S, Jansen M. The metaplastic mosaic of Barrett's oesophagus Virchows Archiv. 2018; (472): 43-54. PubMed PMID: 29500519

6. Harrison R, Perry I, Haddadin W, McDonald S, et al. Detection of intestinal metaplasia in Barrett's esophagus: an observational comparator study suggests the need for a minimum of eight biopsies. Am J Gastroenterol. 2007; (102): 1154-61. PubMed PMID: 17433019.

7. Que J, Garman KS, Souza RF, Spechler SJ. Pathogenesis and

Cells of Origin of Barrett's Esophagus. Gastroenterology. 2019; 157 (2): 349-64. PubMed PMID: 31082367.

8. Theodorou D, Ayazi S, DeMeester SR, Zehetner J, et al. Intraluminal $\mathrm{pH}$ and goblet cell density in Barrett's esophagus. J Gastrointest Surg. 2012; (16): 469-74. PubMed PMID: 22095525 .

9. Sun D, Wang X, Gai Z, Song X, Jia X, Tian H. Bile acids but not acidic acids induce Barrett's esophagus. Int J Clin Exp Pathol. 2015; 8 (2): 1384-92. PMID: 25973022.

10. Hahn HP, Blount PL, Ayub K, Das KM, et al. Intestinal differentiation in metaplastic, nongoblet columnar epithelium in the esophagus. Am J Surg Pathol. 2009; (33): 1006-15. PubMed PMID: 19363439.

11. Bhat $S$, Coleman HG, Yousef F, Johnston BT, et al. Risk of malignant progression in Barrett's esophagus patients: results from a large population-based study. 2011; J Natl Cancer Inst. 103 (13): 1049-57. PubMed PMID: 21680910.

12. Kelty CJ, Gough MD, Van Wyk Q, Stephenson TJ, Ackroyd R. Barrett's oesophagus: intestinal metaplasia is not essential for cancer risk. Scand J Gastroenterol. 2007; 42 (11): 1271-4. PubMed PMID: 17852872.

13. Takubo K, Aida J, Naomoto Y, et al. Cardiac rather than intestinal type background in endoscopic resection specimens of minute Barrett adenocarcinoma. Hum Pathol. 2009; 40 (1): 65-74. PubMed PMID: 18755496. 
14. Watanabe G, Ajioka Y, Takeuchi M, Annenkov A, Kato T, et al. Intestinal metaplasia in Barrett's oesophagus may be an epiphenomenon rather than a preneoplastic condition, and CDX2-positive cardiac-type epithelium is associated with minute Barrett's tumour. Histopathology. 2015; 66 (2): 201-14. PubMed PMID: 25040564.

15. Lavery DL, Martinez P, Gay LJ, Cereser B, et al. Evolution of oesophageal adenocarcinoma from metaplastic columnar epithelium without goblet cells in Barrett's oesophagus. Gut. 2016; 65 (6): 907-13. PubMed PMID: 26701877.

16. Schellnegger R, Quante A, Rospleszcz S, Schernhammer M, et al. Goblet cell ratio in combination with differentiation and stem cell markers in Barrett esophagus allow distinction of patients with and without esophageal adenocarcinoma. Cancer Prev Res. (Phila). 2017; 10 (1): 55-66. PubMed PMID: 27807078.

17. Srivastava A, Golden KL, Sanchez CA, Liu K, et al. High goblet cell count is inversely associated with ploidy abnormalities and

\section{References}

1. Shaheen NJ, Falk GW, Iyer PG, Gerson LB. ACG clinical guideline: diagnosis and management of Barrett's esophagus. Am J Gastroenterol. 2016; 111 (1): 30-50. PubMed PMID: 26526079.

2. Fitzgerald $R C$, di Pietro $M$, Ragunath $K$, Ang $Y$, et al. British Society of Gastroenterology guidelines on the diagnosis and management of Barrett's oesophagus. Gut. 2014; (63): 7-42. PubMed PMID: 24165758.

3. Bennett C, Moayyedi P, Corley DA, DeCaestecker J et al. BOB CAT: A Large-Scale Review and Delphi Consensus for Management of Barrett's Esophagus With No Dysplasia, Indefinite for, or Low-Grade Dysplasia. Am J Gastroenterol. 2015; (110): 662-82. PubMed PMID: 25869390.

4. Montgomery EA, Canto MI, Srivastava A. Evaluation and reporting of biopsies from the columnar-lined esophagus and gastroesophageal junction (GEJ). Ann Diagn Pathol. 2019; (39): 111-7. PubMed PMID: 30802810.

5. Biswas S, Quante M, Leedham S, Jansen M. The metaplastic mosaic of Barrett's oesophagus Virchows Archiv. 2018; (472): 43-54. PubMed PMID: 29500519

6. Harrison R, Perry I, Haddadin W, McDonald S, et al. Detection of intestinal metaplasia in Barrett's esophagus: an observational comparator study suggests the need for a minimum of eight biopsies. Am J Gastroenterol. 2007; (102): 1154-61. PubMed PMID: 17433019.

7. Que J, Garman KS, Souza RF, Spechler SJ. Pathogenesis and Cells of Origin of Barrett's Esophagus. Gastroenterology. 2019; 157 (2): 349-64. PubMed PMID: 31082367.

8. Theodorou D, Ayazi S, DeMeester SR, Zehetner J, et al. Intraluminal $\mathrm{pH}$ and goblet cell density in Barrett's esophagus. J Gastrointest Surg. 2012; (16): 469-74. PubMed PMID: 22095525.

9. Sun D, Wang X, Gai Z, Song X, Jia X, Tian H. Bile acids but not acidic acids induce Barrett's esophagus. Int J Clin Exp Pathol. 2015; 8 (2): 1384-92. PMID: 25973022.

10. Hahn HP, Blount PL, Ayub K, Das KM, et al. Intestina differentiation in metaplastic, nongoblet columnar epithelium in the esophagus. Am J Surg Pathol. 2009; (33): 1006-15. PubMed PMID: 19363439.

11. Bhat S, Coleman HG, Yousef F, Johnston BT, et al. Risk of malignant progression in Barrett's esophagus patients: results from a large population-based study. 2011; J Natl Cancer Inst. 103 (13): 1049-57. PubMed PMID: 21680910. risk of adenocarcinoma in Barrett's esophagus. PLoS One. 2015; 10 (7): e0133403. PubMed PMID: 26230607.

18. Agoston AT, Srivastava A, Zheng $Y$, et al. Prevalence and concordance of subtypes of dysplasia in patients with Barrett's esophagus-associated adenocarcinoma. Mod Pathol. 2014; (27): 162a-162a.

19. Demicco EG, Farris AB, Baba $Y$, Agbor-Etang $B$, et al. The dichotomy in carcinogenesis of the distal esophagus and esophagogastric junction: intestinal-type vs cardiac-type mucosaassociated adenocarcinoma. Mod Pathol. 2011; (24): 1177-90. PubMed PMID: 21572404.

20. Khor TS, Alfaro EE, Ooi EM, Li Y, et al. Divergent expression of MUC5AC, MUC6, MUC2, CD10, and CDX-2 in dysplasia and intramucosal adenocarcinomas with intestinal and foveolar morphology: Is this evidence of distinct gastric and intestinal pathways to carcinogenesis in Barrett esophagus? Am J Surg Pathol. 2012; (36): 331-42. PubMed PMID: 22261707.

12. Kelty CJ, Gough MD, Van Wyk Q, Stephenson TJ, Ackroyd R. Barrett's oesophagus: intestinal metaplasia is not essential for cancer risk. Scand J Gastroenterol. 2007; 42 (11): 1271-4. PubMed PMID: 17852872

13. Takubo K, Aida J, Naomoto $Y$, et al. Cardiac rather than intestinal type background in endoscopic resection specimens of minute Barrett adenocarcinoma. Hum Pathol. 2009; 40 (1): 65-74. PubMed PMID: 18755496.

14. Watanabe G, Ajioka Y, Takeuchi M, Annenkov A, Kato T, et al. Intestinal metaplasia in Barrett's oesophagus may be an epiphenomenon rather than a preneoplastic condition, and CDX2-positive cardiac-type epithelium is associated with minute Barrett's tumour. Histopathology. 2015; 66 (2): 201-14. PubMed PMID: 25040564.

15. Lavery DL, Martinez P, Gay LJ, Cereser B, et al. Evolution of oesophageal adenocarcinoma from metaplastic columnar epithelium without goblet cells in Barrett's oesophagus. Gut. 2016; 65 (6): 907-13. PubMed PMID: 26701877.

16. Schellnegger R, Quante A, Rospleszcz S, Schernhammer M, et al. Goblet cell ratio in combination with differentiation and stem cell markers in Barrett esophagus allow distinction of patients with and without esophageal adenocarcinoma. Cancer Prev Res. (Phila). 2017; 10 (1): 55-66. PubMed PMID: 27807078.

17. Srivastava A, Golden KL, Sanchez CA, Liu K, et al. High goblet cell count is inversely associated with ploidy abnormalities and risk of adenocarcinoma in Barrett's esophagus. PLoS One. 2015; 10 (7): e0133403. PubMed PMID: 26230607.

18. Agoston AT, Srivastava A, Zheng $Y$, et al. Prevalence and concordance of subtypes of dysplasia in patients with Barrett's esophagus-associated adenocarcinoma. Mod Pathol. 2014; (27): 162a-162a.

19. Demicco EG, Farris AB, Baba $Y$, Agbor-Etang $B$, et al. The dichotomy in carcinogenesis of the distal esophagus and esophagogastric junction: intestinal-type vs cardiac-type mucosaassociated adenocarcinoma. Mod Pathol. 2011; (24): 1177-90. PubMed PMID: 21572404

20. Khor TS, Alfaro EE, Ooi EM, Li $Y$, et al. Divergent expression of MUC5AC, MUC6, MUC2, CD10, and CDX-2 in dysplasia and intramucosal adenocarcinomas with intestinal and foveolar morphology: Is this evidence of distinct gastric and intestinal pathways to carcinogenesis in Barrett esophagus? Am J Surg Pathol. 2012; (36): 331-42. PubMed PMID: 22261707. 